PROFESIONALES Y HERRAMIENTAS PARA EL DESARROLLO LOCAL Y SUS SINERGIAS TERRITORIALES. EVALUACIÓN Y PROPUESTAS DE FUTURO IX Coloquio Nacional de Desarrollo Local del GTDL-AGE 

ANTONIO MARTÍNEZ PUCHE, XAVIER AMAT MONTESINOS, ISABEL SANCHO CARBONELL y DANIEL SANCHIZ CASTAÑO (EDS.)

\section{PROFESIONALES Y HERRAMIENTAS PARA EL DESARROLLO LOCAL Y SUS SINERGIAS TERRITORIALES. EVALUACIÓN Y PROPUESTAS DE FUTURO}

IX Coloquio Nacional de Desarrollo Local del GTDL-AGE

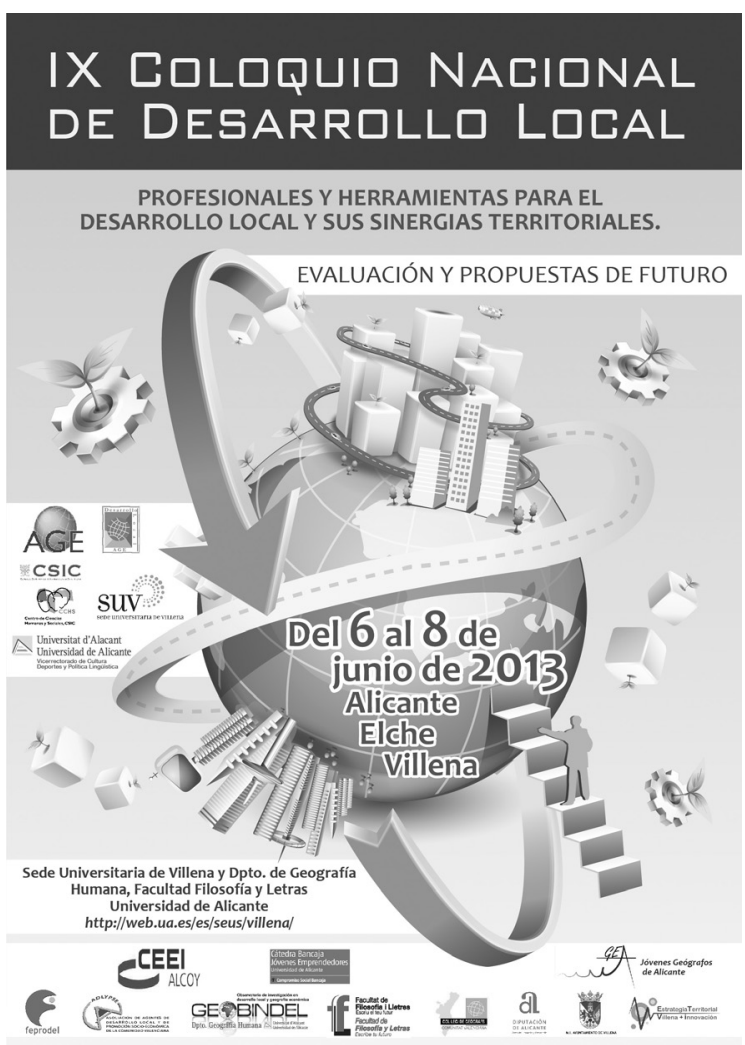


Este libro ha sido examinado y valorado por evaluadores ajenos a la Universidad de Alicante, con el fin de garantizar la calidad científica del mismo.

Publicacions de la Universitat d'Alacant

03690 Sant Vicent del Raspeig

Publicaciones@ua.es

http://publicaciones.ua.es

Telèfon: 965903480

(C) Antonio Martínez Puche, Xavier Amat Montesinos,

Isabel Sancho Carbonell y Daniel Sanchiz Castaño (eds.), 2016

(C) d'aquesta edició: Universitat d'Alacant

ISBN: 978-84-16724-00-0

Dipòsit legal: A 92-2016

Disseny de coberta: candela ink

Composició: Página Maestra (Miguel Ángel Sánchez Hernández)

Impressió i enquadernació: Guada Impresores

\section{unte \\ Unión de Editoriales
Universitarias Españolas \\ WWW.une.es
WWA}

Esta editorial es miembro de la UNE, cosa que garantiza la difusión y comercialización nacional y internacional de sus publicaciones.

Reservados todos los derechos. Cualquier forma de reproducción, distribución, comunicación pública o transformación de esta obra sólo puede ser realizada con la autorización de sus titulares, salvo excepción prevista por la ley. Diríjase a CEDRO (Centro Español de Derechos Repográficos, www.cedro.org) si necesita fotocopias o escanear algún fragmento de esta obra. 


\title{
LA AGENDA LOCAL 21 COMO HERRAMIENTA DE GESTIÓN INTEGRADA EN LOS MUNICIPIOS DE LA PROVINCIA DE ALICANTE (2005-2011). ¿UNA OPORTUNIDAD PERDIDA? ${ }^{1}$
}

\author{
Antonio Martínez Puche \\ Dpto. de Geografía Humana (UA) y Director del Master Oficial DELEITE \\ antonio.martinez@ua.es \\ José Antonio Larrosa Rocamora \\ Dpto. Geografía Humana (UA) \\ JA.Larrosa@ua.es \\ Xavier Amat Montesinos \\ Dpto. Geografía Humana (UA) \\ Xavier.amat@ua.es

\begin{abstract}
"Estos problemas - medioambientales- exigen, en un ámbito global, grandes políticas ambientales; pero es importante hacer hincapié en las acciones realizadas a nivel local, en regiones delimitadas, en la propia casa, entendida como concepto amplio. Se trata de ir dando soluciones concretas a estos problemas, y es en el ámbito local donde pueden ponerse en práctica con mayor facilidad. Conociendo los problemas existentes en un ámbito relativamente pequeño, podremos realizar acciones que intenten corregirlos y que mejoren el entorno" ANCÍN GARCÍA, M., y PARDO, M., (1998): El desarrollo de la conciencia ambiental en las administraciones locales, Fundación Fernando de los Ríos, Madrid, p. 3
\end{abstract}

\section{RESUMEN}

Las agendas locales 21, son instrumentos estratégicos para la gestión integrada de los recursos del territorio. En la provincia de Alicante, se han utilizado de

1 Proyecto GV/2014/085: "Análisis de la innovación territorial, gobernanza y procesos de resiliencia en la Comunidad Valenciana para el favorecimiento de emprendimientos locales. Identificación de instrumentos, evaluación de indicadores y sistematización de buenas prácticas", Conselleria de Educación Cultura y Deporte, Dirección General de Universidades, Estudios Superiores y ciencia. 
una forma partidista y nada integrada. Además, el servicio de la Diputación provincial de Alicante, encargado de realizar e incentivar la Red Municipal de las AL21, junto con su asesoramiento y acompañamiento a los municipios, ha sido reformado. Esto no ha ayudado a favorecer un proceso en el que las AL21, se conviertan en un instrumento funcional y útil para los territorios. La comunicación analiza parte de los resultados del informe que se encargó, por parte de la Diputación Provincial de Alicante, al Departamento de Geografía Humana².

Palabras clave: Sostenibilidad integrada, ayuntamientos, instrumento estratégico, desarrollo local.

\section{THE LOCAL AGENDA 21 AS TOOL OF INTEGRATED MANAGEMENT IN THE MUNICIPALITIES OF ALICANTE (2005-2011). A LOST OPPORTUNITY?}

\section{AbSTRACT}

The local Agenda 21 (AL21), they are strategic instruments for the integrated management of the resources of the territory. In the county of Alicante, they have been used in a partisan way and anything integrated. Also, the service of the provincial of Alicante, in charge of to carry out and to motivate the Municipal Net of AL21, together with their advice and accompaniment to the municipalities, it has been reformed. This has not helped a process AL21, become in a functional and useful instrument for the territories. The communication analyzes the results of an investigation project.

Keywords: Integrated Sustainability, city councils, strategic instrument, local development.

\section{INTRODUCCIÓN}

Las principales líneas de la Estrategia Europea de Desarrollo Sostenible, se trazaron inicialmente en 2001, en la Comunicación de la Comisión, "Desarrollo sostenible en Europa para un mundo mejor: Estrategia de la Unión Europea para un desarrollo sostenible" (COM/2001/264 final). Dicho contenido, se incluyó después en las conclusiones del Consejo Europeo de Gotemburgo de 2001 y formó parte de los trabajos preparatorios de la Unión Europea para la Cumbre mundial de 2002 sobre el desarrollo sostenible (Río + 10) que se celebró en la ciudad sudafricana de Johannesburgo. A este proceso respondió el denominado Documento de Consulta de la Estrategia Española de Desarrollo

2 Informe de sostenibilidad de la provincia de Alicante, en el marco de la implantación de la Agenda 21 Local. Alicante Natura Red Provincial Agenda 21 (2011), dirigido por Antonio Martínez Puche, Departamento de Geografía Humana de la Universidad de Alicante, para el área de Medio Ambiente de la Excelentísima Diputación Provincial de Alicante. 
Sostenible, que fue objeto del informe realizado por el Consejo Económico y Social, (CES 1/2002), y elaborado a solicitud del Ministro de Medio Ambiente. El Consejo Europeo de Bruselas de junio de 2005 reafirmó los objetivos clave del desarrollo sostenible allí trazados. Estos fueron: la protección del medio ambiente, la equidad y la cohesión social, la prosperidad económica, así como el papel de la Unión Europea en este ámbito. Se recordó, asimismo, los principios rectores que debían servir de base para la renovación de la estrategia, basados en el fomento y protección de los derechos fundamentales, solidaridad intra e intergeneracional, garantía de una sociedad abierta y democrática, participación de la ciudadanía, las empresas y los interlocutores sociales, coherencia e integración de las políticas, explotación de los mejores conocimientos disponibles, principios de cautela y en el popular lema de «quien contamina paga».

En diciembre de 2005 se constituye la denominada Red de Redes de Desarrollo Local Sostenible, de la que forma parte el Ministerio de Medio Ambiente, la Federación Española de Municipios y Provincias (FEMP), y el conjunto de Redes provinciales y autonómicas que han trabajado en la implantación de los procesos de Agenda 21 Local. La finalidad de esta red estatal, fue la de constituirse como un foro de debate e intercambio de experiencias entre las distintas redes que, a nivel autonómico y provincial, trabajaban por la Agenda 21 Local con el propósito de promover un concepto de ciudad compacta, compleja, eficiente y cohesionada socialmente. El primer trabajo que desarrolló la Red de Redes de Desarrollo Local Sostenible fue la Estrategia de Medio Ambiente Urbano, aprobada el 15 de junio de 2006. Según el Banco Público de Indicadores Ambientales del Ministerio de Medio Ambiente, en el año 2006 la Red de Redes contaba con 1.901 municipios adheridos y una población total de 19.018.868 habitantes (PRIETO CERDÁN, A. et alii, 2009: 23)³. En esencia, este documento tenía como objetivo general mejorar los resultados medioambientales y la calidad del entorno en las zonas urbanas, garantizando un medio de vida sano para los ciudadanos urbanos europeos, reforzando la contribución del medio ambiente al desarrollo urbano sostenible. Los retos medioambientales a los que se enfrentaban las ciudades europeas tienen importantes repercusiones en la salud y en la calidad de vida de sus habitantes, pero también en el rendimiento económico y en la cohesión social de las ciudades mismas (ELORRIETA, J.I., 2006; 18).

Pero todo ello no es posible sin un verdadero compromiso político y ciudadano que debe participar de un proceso necesitado de reflexión, diálogo, concertación y concreción en acciones y medidas, que superen documentos, que en muchas ocasiones, se quedan en una declaración de buenas intenciones. De ahí la importancia de establecer sistemas de indicadores, no sólo ambientales y de

3 PRIETO CERDÁN, A. et alii, (2009): 12 pasos para la red agenda 21. Nueva metodología para implantar la agenda 21 local, Diputación provincial de Alicante, Alicante, 80 p. 
evaluación integrada de la sostenibilidad, donde entran aspectos como la economía creativa, entornos innovadores, eficiencia energética, paisaje, capacidad para el aprendizaje, talento, etc., que posibilitan la catalogación de territorios inteligentes (FERNÁNDEZ-MACHO, J., GONZÁLEZ CASIMIRO, P., 2009: $55)$.

\section{LAS AGENDAS LOCALES 21, COMO INSTRUMENTOS ESTRATÉGICOS DE PARTICIPACIÓN CIUDADANA Y GESTIÓN SOSTENIBLE}

Es el tiempo de las ciudades, del "piensa globalmente, actúa localmente". Por eso la llave de la sostenibilidad está en las manos de la ciudadanía y de los gobiernos locales, que no localistas, a la hora de actuar de manera efectiva, con acciones concretas en la gestión racional e integrada de los recursos y a la sensibilización y educación de sus ciudadanos, al que se debe unir el compromiso ético, y no sólo estético, de sus políticos ${ }^{4}$. El objetivo de las acciones municipales es el de implicar a la ciudadanía, ya que sin la participación activa de las ciudades, la sotenibilidad se hace imposible ${ }^{5}$. Así se puede establecer en un análisis cartesiano los diferentes niveles de actuación (cuadro 1). Pensar globalmente y actuar globalmente $(A)$, es como actúan Organismos Internacionales como la ONU, o como se trabaja en las grandes cumbres mundiales desde el punto de vista ambiental, de cambio climático o desde el punto de vista monetario, intentando buscar acuerdos globales, que en muchas ocasiones son difíciles de cumplir. Pensar globalmente y actuar localmente (B), es lo que hacen las agencias de desarrollo local, los grupos de acción local o las oficinas 21 cuando gestionan una agenda local 21 , un plan estratégico, una Iniciativa Comunitaria, un proyecto europeo, que se vale de financiación supralocal, pero que intenta activar, poner en valor y favorecer a los recursos locales de una for-

4 "En España, 285 Gobiernos locales (que representan a más de 27 millones de personas) están unidos en la Red de Ciudades por el Clima, una iniciativa que desde 2004 (creada por la Federación de Municipios y Provincias junto al Ministerio de Medio Ambiente) reúne a ciudades comprometidas con el medio ambiente para que compartan experiencias y conocimientos en un foro. El presidente de la red fue a Copenhague -el pasado mes de diciembre- para mostrar algunos de los proyectos en los que se está trabajando. Las ciudades son actores imprescindibles, ya que más de la mitad de ls emisiones provienen de entornos urbanos". Cristina Castro Carbón, "La revolución verde será local", El País, domingo 3 de enero de 2010.

5 "Luis Jiménez, presidente del Observatorio de la Sostenibilidad en España (OSE), afirma que se conseguirá poco globalmente si no hay acción y lucha desde las ciudades. Sobre la capacidad de actuación de los municipios Jiménez opina que se trata de cambiar la cultura urbana, con políticas directas, hay muchas formas racionales y simples de cambiar las cosas, como facilitar a los ciudadanos que puedan reducir los trayectos pequeños en coche (...) A la gente le encanta encontrarse con el alcalde en el autobús, hay cordialidad y facilita el diálogo entre la gente, pero además tiene una efectividad importante, porque se envían señales a la población: todo el mundo puede ir en autobús, se puede romper la cultura del coche". Cristina Castro Carbón, "La revolución verde será local", El País, domingo 3 de enero de 2010. 
ma integrada, generando sinergias. Pensar globalmente y actuar localmente $(C)$, es lo que han hecho algunas superpotencias como EE.UU., provocando y controlando países y conflictos internacionales, a través de sus militares y su economía, para favorecer sus mercados internos, a sus propias multinacionales o bien beneficiarse de la compra-venta de materias primas. Pensar localmente y actuar localmente $(D)$, es lo que hacemos habitualmente las personas, a lo largo de nuestro quehacer diario y cotidiano.

\section{Cuadro 1. Pensar Globalmente, actuar Localmente}

\section{LOCAL $\neq$ LOCALISTA}

Ejemplo:
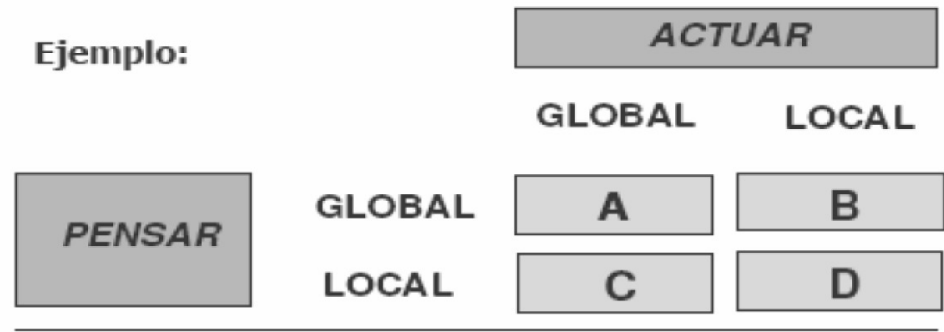
$\mathrm{A}=$ O.N.U., Cumbre de Río, Cumbre de Kyoto, etc.
$\mathrm{B}=$ Agencia de Desarrollo Local, Agendas locales 21,
$\mathrm{C}=\mathrm{EE} . \mathrm{UU}$.
D = Forma en la que actúan normalmente las personas

Fuente: Adaptación David Pérez, $3^{a}$ Edición Master Oficial en Desarrollo Local e Innovación Territorial, Universidad de Alicante, (2009-2010).

En este contexto hay que indicar que la Agenda 21 Local (A21L) es la herramienta diseñada para trabajar por el desarrollo sostenible desde una óptica radicalmente distinta a la tradicional. A pesar del tiempo que lleva en activo (Carta de Aalborg de 1994), bien aplicada, recoge técnicas de diagnóstico integral, más allá de los aspectos meramente ambientales, participando de procedimientos de evaluación y medición de las acciones desarrolladas en los municipios, que ejercen la bien entendida gobernanza.

\subsection{Orígenes de las Agendas Locales 21}

Remontarnos al origen de la sostenibilidad local, significaría viajar en el tiempo hasta el mes de junio del año 1992, y asistir a la Conferencia de Naciones Unidas sobre el Medio Ambiente y Desarrollo, más conocida como Cumbre de la Tierra, llevada a cabo en la ciudad de Río de Janeiro. Para ello se consensuó una declaración de 27 principios (Declaración de Río), y un programa marco 
de actuación, conocido como "Programa 21". El capítulo 28 del Programa 21, trata íntegramente de las iniciativas de las autoridades locales en apoyo del mismo, constituyendo los cimientos de la "sostenibilidad local" y el origen de las Agendas 21 locales (PRIETO CERDÁN, A., 2009: 19). Pero será en 1994 en la ciudad danesa de Aalborg, donde 80 autoridades locales europeas firmen la "Carta de las Ciudades y Pueblos Europeos para la Sostenibilidad", celebrada en esta ciudad durante los días 24 a 27 de mayo. En total asistieron 330 participantes y aquí se inició la campaña Europea de Ciudades y Poblaciones Sostenibles, que han tenido hasta la fecha siete cumbres internacionales (cuadro 2). Este programa quiere reconsiderar el desarrollo desde los nuevos valores de la sostenibilidad, recuperando la capacidad de interpretación y formulación de objetivos con respecto a los espacios urbanos y su entorno más inmediato. Ello obliga necesariamente a compatibilizar el medio ambiente, la economía y las necesidades sociales, dando lugar al establecimiento de perspectivas estratégicas integrales (PRATS, F., 1996: 97).

\section{Cuadro 2. Conferencias de Pueblos y Ciudades Sostenibles}

\begin{tabular}{|c|c|}
\hline Conferencia & $\begin{array}{l}\text { Principales conclusiones en materia de gobernanza y } \\
\text { ciudadanía }\end{array}$ \\
\hline $\begin{array}{l}\text { Primera } \\
\text { Aalborg }\end{array}$ & $\begin{array}{l}\text { - Los ciudadanos se hacen co-responsables del bienestar } \\
\text { humano y de la naturaleza } \\
\text { - Las ciudades reconocen un papel importante en el cambio } \\
\text { de los modos de vida, de producción y de distribución en } \\
\text { el espacio } \\
\text { - Las ciudades reconocen que cada una es característica } \\
\text { - Las ciudades se comprometen a resolver sus propios pro- } \\
\text { blemas bajo el principio de concertación } \\
\text { - Las ciudades se comprometen a concertar planes de ac- } \\
\text { ción locales }\end{array}$ \\
\hline $\begin{array}{l}\text { Segunda Conferencia. } \\
\text { Lisboa (1996) }\end{array}$ & $\begin{array}{l}\text { - Las autoridades locales deben facilitar, dinamizar y lide- } \\
\text { rar el proceso de AL21 hasta que el resto de agentes desa- } \\
\text { rrollen el impulso necesario } \\
\text { - Las autoridades locales deben promover redes de agentes } \\
\text { comprometidos en el gobierno y la gestión municipales }\end{array}$ \\
\hline $\begin{array}{l}\text { Tercera Conferencia. } \\
\text { Hannover (2000) }\end{array}$ & $\begin{array}{l}\text { - Se insta a la Comunidad internacional a que apoye el au- } \\
\text { togobierno local (autonomía financiera) } \\
\text { - Se insta a los gobiernos nacionales a crear marcos políti- } \\
\text { cos de apoyo al desarrollo sostenible } \\
\text { - Se insta a los gobiernos nacionales a reconocer a las ciu- } \\
\text { dades como interlocutores válidos para la realización de } \\
\text { proyectos de desarrollo internacional sostenible }\end{array}$ \\
\hline
\end{tabular}




\begin{tabular}{|c|c|}
\hline $\begin{array}{l}\text { Cuarta Conferencia. } \\
\text { "Inspiración para el } \\
\text { futuro" Aalborg } 2004\end{array}$ & $\begin{array}{l}\text { - Se aboga por el impulso a la democracia participativa } \\
\text { (proceso de toma de decisiones abierto, transparente y } \\
\text { responsable) } \\
\text { - Se aboga por la cooperación con otras entidades locales y } \\
\text { otros niveles de gobierno } \\
\text { - Se aboga por una gestión municipal sostenible (procesos } \\
\text { de sostenibilidad local en la propia gestión municipal } \\
\text { transversales a todas las áreas municipales) }\end{array}$ \\
\hline $\begin{array}{l}\text { Quinta Conferencia. } \\
\text { "Llevando el } \\
\text { compromiso a las } \\
\text { calles". Sevilla, } 2007\end{array}$ & $\begin{array}{l}\text { - Se reconoce la falta de relación entre autoridades, técni- } \\
\text { cos municipales y ciudadanos }\end{array}$ \\
\hline $\begin{array}{l}\text { Sexta Conferencia. } \\
\text { Dunkerque (Francia, } \\
\text { 2010) }\end{array}$ & $\begin{array}{l}\text { La } 6^{\text {a }} \text { Conferencia Europea de Ciudades Sostenibles fue una } \\
\text { oportunidad para compartir y beneficiarse de las experien- } \\
\text { cias sostenibles entre ciudades y territorios europeos. Sus } \\
\text { objetivos principales: } \\
\text { - Evaluar los avances en cuanto a desarrollo sostenible des- } \\
\text { de hace } 15 \text { años } \\
\text { - Emprender una reflexión política y estratégica sobre las } \\
\text { oportunidades que ofrece el desarrollo sostenible } \\
\text { - Explorar diferentes ámbitos de acción de los gobiernos } \\
\text { locales que permitan contribuir con el desarrollo soste- } \\
\text { nible } \\
\text { - Examinar el modo en que los gobiernos locales pueden } \\
\text { implicar a sus ciudadanos en comportamientos sosteni- } \\
\text { bles } \\
\text { - Analizar las evoluciones derivadas de los procesos políti- } \\
\text { cos a nivel europeo } \\
\text { - Concluir con una reflexión sobre las próximas acciones } \\
\text { de la Campaña Europea de Ciudades Sostenibles. }\end{array}$ \\
\hline $\begin{array}{l}\text { Séptima. Ginebra } \\
\text { (Suiza, 2013) }\end{array}$ & $\begin{array}{l}\text { - Se ratifica la sostenibilidad en todas las áreas de la ad- } \\
\text { ministración pública, intentando identificar mecanismos } \\
\text { institucionales efectivos para combatir las crisis financie- } \\
\text { ra y ambiental actuales. } \\
\text { - Se apuesto por un sistema de desarrollo actual y la ne- } \\
\text { cesidad de un cambio de paradigma económica para fa- } \\
\text { vorecer un desarrollo sostenible. Hay que fomentar una } \\
\text { economía solidaria y acometer el modelo dominante. En } \\
\text { este sentido las ciudades cumplen un rol importante (ciu- } \\
\text { dades en transición). La creciente presión hacia los recur- } \\
\text { sos naturales, provoca más desequilibrios que afectan a } \\
\text { poblaciones vulnerables. De ahí que se tenga que apostar } \\
\text { por soluciones creativas, responsables y solidarias. }\end{array}$ \\
\hline
\end{tabular}

Fuente: Carrasco Monteagudo, C. (2008: 206) y Elaboración propia. 
La utilización de herramientas en materia de gestión ambiental integrada, así como el mayor compromiso (político y ciudadano) frente a temas como el desarrollo sostenible, la educación ambiental y el trabajo en red, han tenido mayor predicamento en la última década en todos los niveles de la sociedad. En este sentido, destacan las red de ciudades y pueblos sostenibles de Castilla-La Mancha (2002), Udalsearea 21 red vasca de municipios hacia la sostenibilidad (2002), Xarxa balear de sostenibilitat (2002) y la Xarxa de Ciutats i Pobles cap a la sostenibilitat de la Diputación de Barcelona (1997) y la Red provincial de Agendas Locales 21 de la provincia de Alicante (2005), entre otras (PRIETO CERDÁN, A., et alii, 2009: 13).

\subsection{La Red provincial de Agendas Locales 21 de Alicante}

Antes de la constitución de la red de Agendas Locales 21 de la provincia de Alicante, algunos ayuntamientos, vinculados a las actividades del turismo, urbanismo y la industria, se habían adscrito a la carta de Aalborg, y habían comenzado a trabajar en la aplicación metodológica de la Agenda Local 21 (MARTÍNEZ PUCHE, A., et alii, 2008: 230) .

El 3 de febrero de 2005, y a iniciativa de la Diputación Provincial de Alicante y tras acuerdo plenario, se aprueba la constitución de la red provincial, con la suscripción a los compromisos de Aalborg y a la Carta Provincial ("Carta de Catî"), firmada el 13 de octubre en un hotel en el término municipal de Petrer, propiedad de la Diputación Provincial (Xorret de Catí). Este fue el marco de referencia que estableció el inicio, ámbito y objetivos de la Red Provincial Agenda 21 de Alicante, que comenzó su andadura con 41 municipios, para pasar en 2007 a 48, en 2008 a 64 municipios, en 2009 a 75, y que en 2010 tuvo un total de 78, de los 141 municipios de la provincia de Alicante.

La coordinación y liderazgo de la Red correspondía al Área de Medio Ambiente de la Diputación de Alicante y sus objetivos y funciones eran:

- Fomentar la elaboración de las Agendas 21 en los municipios de Alicante.

- Favorecer el intercambio de información y experiencias entre los miembros de la misma para alcanzar la meta del desarrollo sostenible.

- Establecer programas que incrementen el conocimiento de los ciudadanos sobre el desarrollo sostenible.

- Colaborar con otras campañas, asociaciones y redes que tengan como fin la sostenibilidad.

6 Así, hasta el año 2002 existían un total de 14 municipios adscritos a la carta de Aalborg, y que habían comenzado a realizar la fase de diagnóstico ambiental. Destacan los ayuntamientos de Agost, Alcoi, Elche, Elda, Muro de Alcoi y Petrer, entre los municipios industriales. Por otra parte, destacan los de Alicante, Benidorm, Denia, Calpe, Santa Pola, Teulada-Moraira, Benifato, Jalón y la Mancomunidad de la Vega Baja, localizada en Bigastro. 
En cuanto a la estrategia de apoyo a los municipios, ésta consistió en el establecimiento de convenios que sirvieran de base para la elaboración de los planes de acción local, para lo cual se creó la Oficina 21, que tenía por objeto centralizar toda la información y experiencias que se fueran desarrollando.

Durante la primera semana del mes de junio de 2008, se realizó en el hotel del Xorret de Catí, unas jornadas de convivencia bajo el título de "Otras miradas sobre la agenda local 21”. Estas jornadas, que duraron día y medio, fueron realizadas tras meses de trabajo y análisis cuantitativos, realizados por parte de la consultora madrileña Pangea, expertos en conflictos medioambientales (Fotos 1 y 2). El motivo de este estudio, fue analizar el porqué la agenda 21 local no funcionaba como una red de municipios, y porqué era percibida como una herramienta, que sólo se quedaba en un diagnóstico ambiental del municipio, que naturalmente era subvencionado por parte de la Diputación ${ }^{7}$.

Sin lugar a dudas, las agendas 21 locales habían perdido dinamismo y había que apostar por estrategias adaptadas a las necesidades reales del territorio. En ello influye la nueva cultura del territorio y de su ordenación, donde las Agendas Locales 21 deben tener un papel estratégico a medio y largo plazo. A su vez, hay que tener en cuenta que no existen "recetarios" que garanticen el éxito, ya que cada realidad territorial responde a unas necesidades individualizadas que se tienen que conocer "in situ", y a las que no se debe considerar bajo el prisma de "patrones" homogéneos. Otro de los elementos que se evidenciaba en la provincia de Alicante, era que las AL21 debían responder a un proceso que conllevara un consenso (político y ciudadano) y una reflexión participativa, donde previamente se debía contestar a las preguntas del porqué, cómo, cuándo, para qué y con quien para poder alcanzar un enfoque integrado. Otras de las evidencias que no se cumplían, fue la posibilidad que tienen las AL21 para prever un modelo territorial, a través de la planificación de escenarios (tendencial, sostenible y actual), que las dota de dinamismo y que obliga a trabajar en red. Por último, se evidenció la importancia del contexto y la cultura local, para

7 Entre los aspectos que destacó la consultora destacan los siguientes: La agenda local 21, había sido la principal herramienta para dinamizar la participación de los ayuntamientos; difícil relación entre los cargos ejecutivos y el personal técnico; necesidad de mayor intercambio entre la agenda local 21 y los instrumentos de gestión ambiental de los ayuntamientos. Por otra parte, la mayoría de ayuntamientos de la red, consideraban que la Agenda Local 21, era una herramienta que podía ser mucho más eficaz de lo que era, ya que afirmaban que la AL21 debía transmitir entusiasmo y capacidad de cambio, utilizando el término "buen rollo" para caracterizar las relaciones entre el área ambiental de la diputación provincial y los ayuntamientos de la red; echaban en falta la consideración singularizada de cada uno de los municipios de la red, que respondían a un contexto diferenciado, con problemas diversos y necesitados de adaptaciones flexible de los criterios de la AL21; se apostaba porque la AL21, fuera también un instrumento que pusiera en valor aquellos recursos singulares que entroncaban con la identidad; según la gran mayoría, la AL21 debía preparar a los municipios y anticiparlos a las resistencias al cambio, superando los miedos que se generan, ante algunas situaciones y posiciones inmovilistas. 
que la Agenda Local 21, resultara exitosa. De ahí la importancia de la participación social y ciudadana proactiva, ya que no es lo mismo asistir que participar.

Al cambio de metodología de trabajo en 2009, (PRIETO CERDÁN, A. et alii, 2009: 51), se unió el análisis de la situación de las agendas locales 21, que se llevó a cabo por parte del departamento de Geografía Humana de la Universidad de Alicante en 2010, bajo el título de Informe de sostenibilidad en el marco de la implantación de la agenda local 21. Alicante Natura Red Provincial Agenda 21, cuya situación de partida podemos resumir en el análisis DAFO (Cuadro 3).
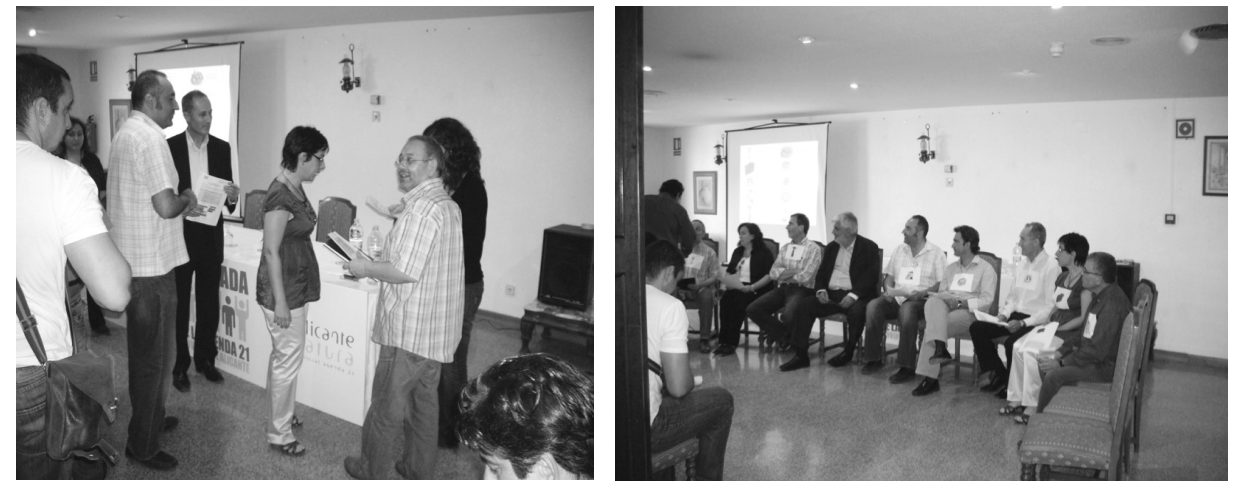

Imágenes 1 y 2: Dinámica participativa en "Otras miradas sobre la AL21".

Fuente: Antonio Martínez Puche.

En él se observa cómo la AL21, muchas veces era utilizada como un instrumento político, donde los ayuntamientos se adscribían sin tener un sentido global y de proceso, y tan sólo por valerse de la subvención de la auditoría ambiental. Por otra parte, se evidenció la falta de recursos humanos cualificados y la vinculación entre la continuidad de las fases de la AL21, y el grado de compromiso político de los ayuntamientos. Otro de los aspectos destacados fue la falta de permeabilidad del objetivo general de la AL21 entre los ciudadanos, que había provocado que los foros ciudadanos no cumplieran su papel de interactuar con las labores técnicas y las decisiones políticas. Eso redundaba en una simplificación en cuanto a lo que se entendía por una Agenda Local 21 (AL21), que sólo se observaba como un instrumento descriptivo, de diagnóstico, y no como una herramienta de planificación estratégica, complementaria a otras acciones sectoriales realizadas en el municipio. 
Cuadro 3. Análisis Dafo. Red Agenda Local 21, (2005-2010)

\begin{tabular}{|c|c|}
\hline DEBILIDADES & AMENAZAS \\
\hline $\begin{array}{l}\text { - Primar la calidad frente a la cantidad. } \\
\text { - Simplificación de la AL21, con al fase de } \\
\text { diagnóstico. } \\
\text { - Asimilación de la AL21 como un simple } \\
\text { proceso mecánico y poco interactivo. } \\
\text { - Confundir asistencia con participación. } \\
\text { - Lo sencillo (comprensible y asumible por } \\
\text { todos), frente a lo simple (diagnóstico e in- } \\
\text { dicadores). } \\
\text { - Falta de instrumentos que legitimen la } \\
\text { participación "proactiva". } \\
\text { - Falta del proceso bottom up. Se aplica de } \\
\text { arriba abajo (top dwon). }\end{array}$ & $\begin{array}{l}\text { - Hastío. Mucha teoría y falta de concre- } \\
\text { ción y logros. La falta de acción, nos lleva } \\
\text { a una falta de reacción. } \\
\text { - Cuando se acaba la subvención, se acaba } \\
\text { el interés. } \\
\text { - Plantear la AL21, como un proceso a cor- } \\
\text { to plazo. Miras temporales cortas, logros } \\
\text { limitados. } \\
\text { - Falta de interés político versus excesivo } \\
\text { interés político. } \\
\text { - La utilización interesada y sesgada, sólo } \\
\text { nos lleva a una política de fachada "efecto } \\
\text { corporación dermoestética". } \\
\text { - Compromiso político, pero falta de } \\
\text { RR.HH., (cualificados) y recursos econó- } \\
\text { micos (la continuidad supeditada a la fi- } \\
\text { nanciación). } \\
\text { - Asimilar, simplificando, la aplicación de } \\
\text { la AL21 con la adscripción a la red provin- } \\
\text { cial. }\end{array}$ \\
\hline FORTALEZAS & OPORTUNIDADES \\
\hline $\begin{array}{l}\text { - Mucha información del proceso y del } \\
\text { procedimiento en la web. } \\
\text { - Soporte web y herramientas informáticas. } \\
\text { Facilidad para participar de forma virtual } \\
\text { en el proceso. } \\
\text { - Existencia de una red provincial, articula- } \\
\text { da, con recursos (económicos como huma- } \\
\text { nos), técnicas e instrumentos. } \\
\text { - Lo interactivo frente a lo aséptico. } \\
\text { - Compromiso voluntario y político de los } \\
\text { ayuntamientos. } \\
\text { - La posibilidad de establecer las AL21 en } \\
\text { un marco de buenas prácticas sostenibles. }\end{array}$ & $\begin{array}{l}\text { - Establecer una plataforma virtual, más } \\
\text { interactiva y propiciadora de foros interac- } \\
\text { tivos para tratar problemáticas comunes, y } \\
\text { posibles soluciones. Foros intermunicipa- } \\
\text { les virtuales. } \\
\text { - Foros intermunicipales presenciales. Pro- } \\
\text { piciar el encuentro, el intercambio de expe- } \\
\text { riencias, pero en aquellos municipios que } \\
\text { han hecho gala de resultados evidentes. } \\
\text { - Foros intraRedes. Establecer estrategias } \\
\text { de comunicación y actuación con otras re- } \\
\text { des de trabajo AL21. } \\
\text { - Superar el concepto y uso tradicional de } \\
\text { la AL21, y aplicarla como herramienta téc- } \\
\text { nica y polivalente para establecer acciones } \\
\text { estratégicas o integrarlas en las Memorias } \\
\text { de los PGOU. Definir el modelo de desa- } \\
\text { rrollo de nuestro término municipal. }\end{array}$ \\
\hline
\end{tabular}

Fuente: Elaboración propia. 


\subsection{Informe de sostenibilidad en el marco de la implantación de la Agenda} Local 21 de la Provincia de Alicante

En la presente investigación fue importante la constatación empírica, cuyos resultados más significativos se habían basado en encuestas, estructuradas en diez preguntas, con respuestas multivariables y articuladas en cuatro bloques. Así, se pasaron estas encuestas a todos los municipios de la provincia de Alicante, independientemente de que pertenecieran o no a la Red Provincial Agenda 21. De esta forma se intentó validar la operatividad de la A21L y la fase en la que se encontraban (preauditoría, auditoría y Plan de Acción Local) (Imagen 1). Por otra parte, se intentó identificar los instrumentos y herramientas de gestión ambiental que al margen de la A21L, se estaban aplicando en los municipios. También, las actuaciones más interesantes que en materia de sostenibilidad estaban aplicando los ayuntamientos, y que fueron desde campañas de información y sensibilización ciudadana (niños, amas de casa, empresarios, jubilados, etc.), hasta la formación y especialización en materia de medio ambiente, edición de manuales y boletines de información o inversiones en políticas e instrumentos para el fomento de políticas y proyectos medioambientales.

Estas encuestas sobre sostenibilidad municipal e integrada, comprendían cuatro bloques cuya finalidad era la que sigue ${ }^{8}$. El primer bloque tenía como objetivo obtener información sobre las actuaciones, instrumentos y acciones vinculadas con la sostenibilidad, ya fuera desde el marco de las A21L o en relación a otros elementos de gestión, como puedan ser las concejalías de Medio Ambiente, los consejos municipales de medio ambiente, etc. El segundo bloque pretendía analizar los ámbitos de actuación dónde se aplican las políticas de sostenibilidad en los municipios de la provincia. Debido a la polarización social, demográfica, económica de los municipios, los ámbitos de aplicación de las políticas de sostenibilidad podían variar, de ahí que fuera fundamental conocer el contexto del área y elaborar políticas actuación desde la base. El tercero de los bloques, integraba cuatro preguntas, en la que se pretendía analizar los medios técnicos y recursos humanos vinculados a la aplicación, control, participación ciudadana y seguimiento de las políticas de sostenibilidad. Por último, el cuarto bloque, se correspondía con una pregunta que tenía como objetivo principal cuantificar y cualificar los problemas ambientales existentes en el municipio, con el fin de conocer la realidad ambiental del mismo.

8 Ficha técnica de la encuesta. Ámbito Territorial: Municipios de la Provincia de Alicante.

- Población de Estudio: 141 ayuntamientos de los municipios provincia de Alicante.

- Tamaño de la muestra: 105 encuestas que representan el 75\% del total de municipios de la provincia de Alicante. 78 Municipios con Agendas 21 Locales, de los que 66 pertenecen a la red provincial.

- Metodología: Cuestionario cumplimentado mediante entrevista telefónica, correo electrónico, y/o presencial. Se contactaron con todos los municipios de la provincia de Alicante.

- Fecha de realización: Noviembre y Diciembre de 2010. 


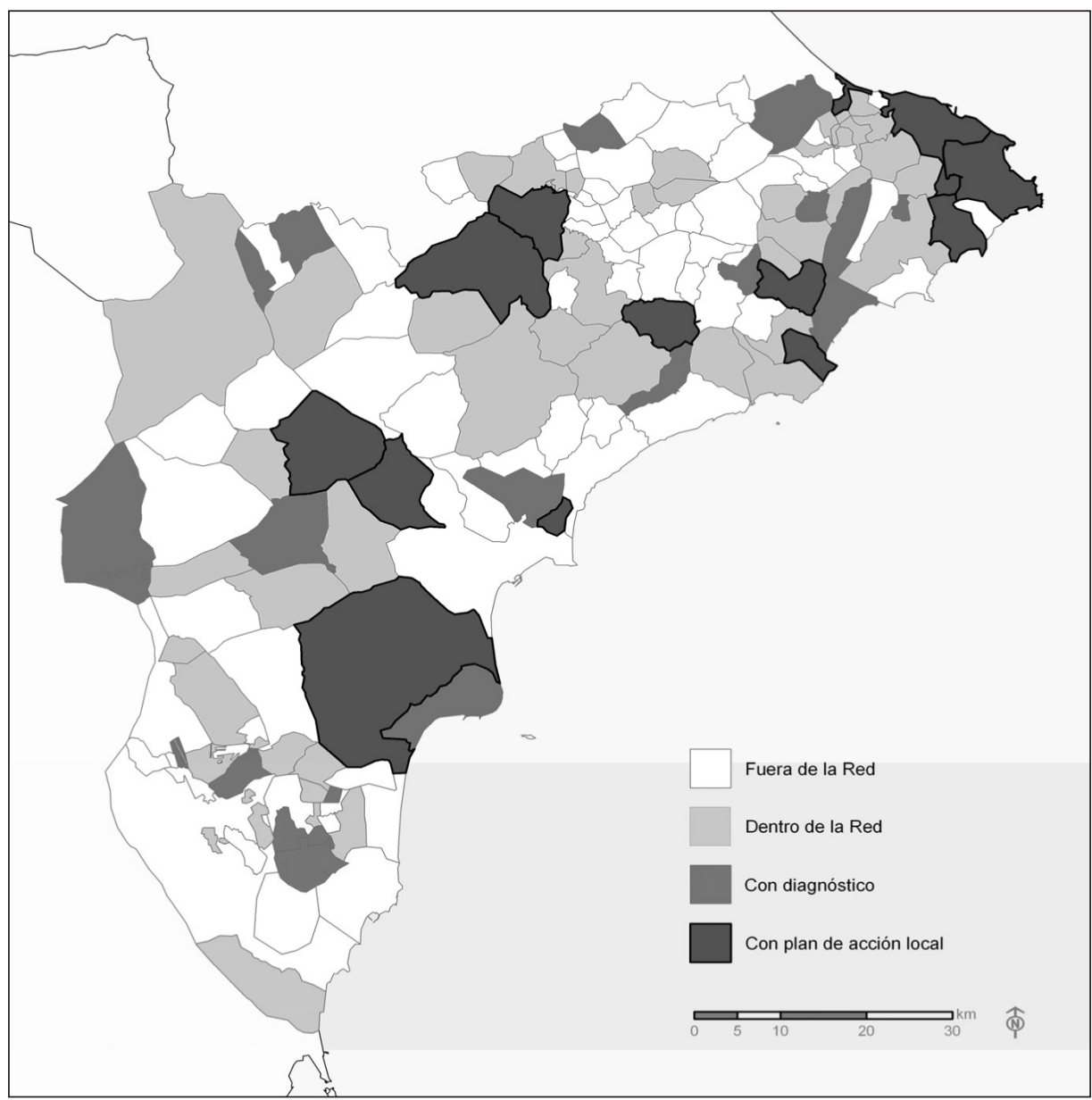

Imagen 1. Situación en la aplicación de la Agenda Local 21. Municipios de la Provincia de Alicante, 2011.

Fuente: Elaboración Propia.

En la encuesta fue interesante resaltar los ámbitos municipales en los que se había aplicado las políticas de sostenibilidad (medio urbano, medio rural, espacios naturales, etc.), el grado de aplicación del Plan de Acción Local (PAL) y los instrumentos de seguimiento para controlar y auditar la información ambiental de los municipios (indicadores, memorias anuales, foros de ciudadanos, etc.). En este sentido, y por parte de los técnicos medioambientales, agentes de desarrollo local y políticos locales implicados en la contestación de las encuestas, se trataron de identificar y especificar los problemas más urgentes a paliar en el municipio, y que según las distintas opiniones, era más importante 
atajar en pos de la mejora de una sostenibilidad integrada y calidad del entorno vital (contaminación acústica, olores, residuos urbanos, residuos industriales y agrícolas, tratamiento de aguas, espacios naturales, infraestructuras y equipamientos para peatones y bicicletas, tráficos rodado, etc.).

Los resultados de las encuestas fueron complementados con el análisis de indicadores transversales para valorar los aspectos que inciden de manera notable en el desarrollo sostenible de un municipio. De ahí que de todos los municipios de la provincia de Alicante, y atendiendo a diversas fuentes, se analizaron indicadores sociodemográficos y económicos; indicadores de usos de suelo y planeamiento urbano; indicadores de biodiversidad; e indicadores de cultura (Cuadro 4).

De los 105 municipios que respondieron la encuesta, 71 confirmaron que tenían A21L. De éstos, el 38\% había finalizado su auditoría, el 35\% tenía aprobado el Plan de Acción Local (PAL) y un 14\% habían dado por concluida la preauditoría. A su vez, entre las acciones más destacadas estaban las campañas de sensibilización ambiental desarrollada en colegios (70\%) y dirigida a la ciudadanía en general (70\%). No obstante, las campañas de sensibilización llevadas a cabo hacia otros colectivos, como empresarios locales, habían sido menos incisivas (32\%), echándose en falta la elaboración de guías técnicas para la implantación y el desarrollo de políticas ambientales, que sólo se cifraba en un $18 \%$ de los casos. Por el contrario, las acciones formativas tuteladas por la Diputación, y dirigidas a los técnicos y políticos en materia de medio ambiente y sostenibilidad, habían sido muy valoradas por los ayuntamientos (el $43 \%$ lo mencionaba), al igual que las inversiones para acciones de medio ambiente y sostenibilidad, junto con las de difusión e información en estas materias (señalado por el $60 \%$ de los ayuntamientos).

Los ayuntamientos de la provincia de Alicante concedían un gran valor a la educación ambiental en los colegios, la elaboración de manuales de buenas prácticas en materia de sostenibilidad, la formación de técnicos y políticos; y, sobre todo, el incremento de las inversiones para acciones de medio ambiente y sostenibilidad. También se desprendía de los resultados de las encuestas, la necesidad de que los ayuntamientos se dotaran de plazas de técnicos para realizar labores ligadas a la gestión medioambiental y programas de desarrollo sostenible. Sólo el 24\% tenía un técnico y el $5 \%$ más de dos. También fue importante la aplicación de los PAL, que en el último año habían experimentado un notable desarrollo, aunque en general sólo un 30\% de los ayuntamientos tenían PAL. Los indicadores medioambientales se habían destacado como la primera herramienta de seguimiento de los programas y las acciones municipales en materia de medio ambiente, como así confirmaron más del 50\% de los ayuntamientos, en detrimento de otras herramientas como la memoria anual de sostenibilidad (16\%) o el foro de ciudadanos (32\%). En este sentido, la participación ciudadana debería de cultivarse más en los municipios alicantinos, ya 


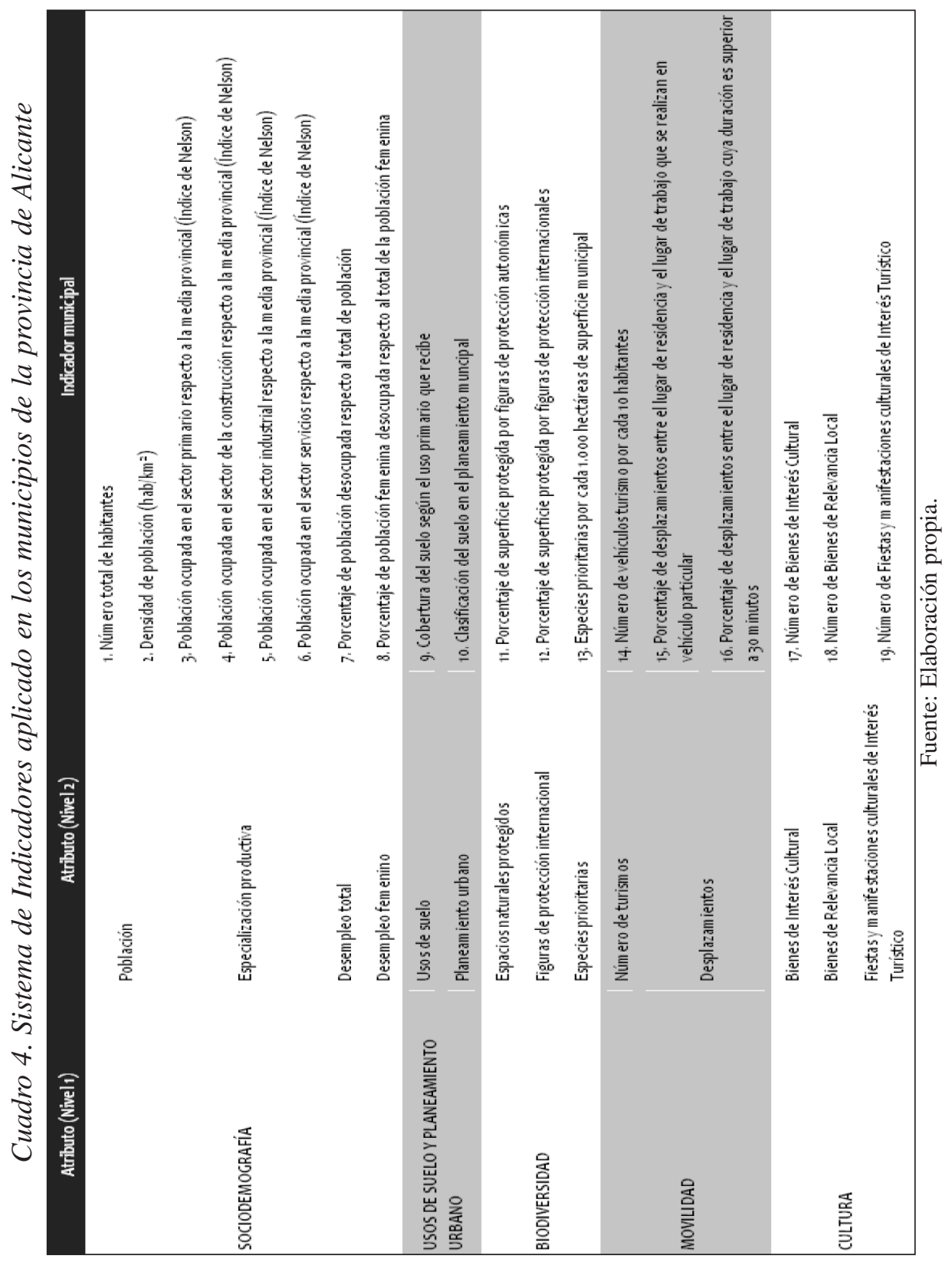


que sólo el 26\% de los ayuntamientos afirmaron que los habían convocado con cierta regularidad.

Finalmente, se pudieron testar cuáles eran los problemas ambientales más acuciantes y cuáles los menos importantes en los municipios de la provincia. La contaminación acústica y por olores eran los menos importantes, según la valoración de más del $45 \%$ de los encuestados. Se mostró un interés mayor, aunque moderado, hacia los problemas ligados al tratamiento de residuos urbanos, industriales y agrícolas, la gestión urbanística, el tratamiento de aguas residuales, la gestión de espacios naturales y la carencia de viales para peatones y bicicletas.

\section{Conclusiones}

La Agenda Local 21 de la Provincia de Alicante no ha sido ni operativa ni estratégica (integrada), en la mayoría de los municipios en los que se ha aplicado. De hecho, a finales de 2011 desapareció la red provincial, ante los ajustes realizados en el área de medio ambiente de la Diputación Provincial de Alicante, que provocó un ajuste en las prioridades de gestión medioambiental hacia los municipios, por parte del organismo provincial. Así, se apostó por las materias de eficiencia energética, y se abandonó como elemento estratégico, el apoyo a las agendas locales 21 , hecho que no ha contribuido a que muchos ayuntamientos hayan continuado con el procedimiento y la metodología establecida ya por la Diputación en su manual de 12 pasos para la red agenda 21. Nueva metodología para implantar la agenda 21 local (PRIETO, 2009).

Por otra parte, hay que significar que la estética ha primado sobre la ética, y que en cuanto a los temas ambientales municipales, ha sido más evidente la pose frente al poso. Las agendas locales 21 , se han concebido desde una perspectiva finalista y utilitarista, por parte de la mayoría de los ayuntamientos de la provincia de Alicante, y en muchas ocasiones, la intención de continuidad en el tiempo ha sido inexistente, primando el proyecto (corto plazo) más que el proceso (largo tiempo e integrado en las acciones municipales).

Además, hemos evidenciado que el mayor grado de inversión pública a través de subvenciones (fase de diagnóstico o premio al municipio sostenible) no ha garantizado un incremento de mayor concienciación medio ambiental, ni un mayor compromiso por la sostenibilidad, ni por parte de los ayuntamientos, ni por parte de la ciudadanía en su conjunto. Tampoco en cuanto a la habilitación de personal técnico municipal dedicado a estas temáticas. Se han realizado campañas de sensibilización ambiental por parte de la Diputación, en centros de formación y educativos. También se estableció el premio de municipio sostenible, para premiar y gratificar a aquellas poblaciones de la provincia, que habían desarrollado acciones en materia de sostenibilidad. Pero desaparecido el servicio encargado de la gestión de estas acciones e incentivos (Oficina 21 
y su personal), y acabado el dinero para las subvenciones que primaban estas actividades, ha finalizado el proyecto, sin generar un tejido realmente concienciado con las problemáticas ambientales de los municipios, que por otra parte, calificaron de "poco importantes", en la mayoría de los casos analizados en las encuestas de nuestro informe.

Por último, significar que las acciones de sostenibilidad municipal que muchos ayuntamientos han llevado a cabo, ha carecido de un enfoque integrado (ambiental, cultural, social, económico, político, ético, etc.). En efecto, ha habido actividades, infraestructuras, medidas e inversiones, que han tenido un notable carácter sectorial y finalista, vinculadas a la gestión de residuos, a la conservación ambiental, a la mejora de equipamientos, que en su conjunto han respondido a necesidades puntuales y accesorias. Estas han carecido de una verdadera concienciación y sensibilización vinculada a la aplicación de la sostenibilidad municipal, como se establece en la deontología y acciones de las agendas locales 21 . De ahí, que no se haya fomentado la participación ciudadana (foro ciudadano) ni la evaluación, ni las auditorias ambientales (a través del PAL), y que no se hayan implementado acciones más allá de las perentorias, para la gestión del agua, residuos, ruidos, parajes naturales, etc. Sin duda, hubiera sido una gran oportunidad para los ayuntamientos, el que se hubieran concebido las Agendas Locales 21, como instrumentos estratégicos, integrados e inclusivos, para la gestión de todos los recursos municipales, con la clara finalidad de mejorar la calidad de vida, en su conjunto, de la ciudadanía.

\section{BiBLIOGRAFÍA}

ANCIN GARCÍA, M. y PARDO, M. (1998): El desarrollo de la conciencia ambiental en las administraciones locales, Fundación Fernando de los Ríos, Madrid, $32 \mathrm{p}$.

AA.VV. (2007): El desarrollo urbano sostenible en el periodo de programación 2007-2013, Grupo de trabajo de Medio Ambiente Urbano y Fondos Comunitarios, Red de autoridades ambientales, Ministerio de Medio Ambiente, mecanografiado, Madrid, $38 \mathrm{pp}$.

BARRUTIA, J. M.; AGUADO, I. y ECHEVARRIA, C. (2007): "Networking for Local Agenda 21 implementation: Learning fromexperiences with Udaltalde and Udalsarea in the Basque autonomous community", Geoforum, $\mathrm{n}^{\circ}$ 38, pp. 33-48.

CARRASCO MONTEAGUDO, I.(2002): "Innovación institucional y participación ciudadana. El caso de la Agenda Local 21”, Economía Industrial, nº368, Madrid, pp. 205-212.

DONOLO, C. (2003): Il distretto sostenibile, Governare i beni comuni per lo sviluppo, editorial FrancoAngeli, Milano, 238 pp.

ELORRIETA, J.I. (2006): "Estrategia Temática Europea sobre Medio Ambien- 
te Urbano. La batalla crucial por la sostenibilidad", Ambienta, Ministerio de Medio Ambiente, Madrid, pp. 18-23.

ESTEVAN, A. (1996): "Actividades industriales y buenas prácticas", Primer catálogo español de buenas prácticas, vol. I, Ministerio de Obras Públicas, Transporte y Medio Ambiente, Madrid, pp. 111-123.

FALUDI, A. (2007): "Método Abierto de Coordinación en planificación territorial a escala de Unión Europea", en Farinós, J. y Romero, J. (eds.), Territorialidad y buen gobierno para el desarrollo sostenible. Nuevos principios y nuevas políticas en el espacio europeo, Publicaciones Universidad de Valencia, Valencia, pp. 77-100.

FARINÓS, J., ROMERO, J. (eds.) (2007): Territorialidad y buen gobierno para el desarrollo sostenible. Nuevos principios y nuevas políticas en el espacio europeo, Universidad de Valencia, Valencia, $258 \mathrm{pp}$.

FARINÓS DASI, J. (2008): "Gobernanza territorial para el desarrollo sostenible: estado de la cuestión y agenda" en Boletín de la Asociación de Geógrafos Españoles, $\mathrm{n}^{\circ}$ 46, Murcia, pp. 11-32.

FERNÁNDEZ-MACHO, J., GONZÁLEZ CASIMIRO, P. (2009): Evaluación de territorios inteligentes en la sociedad del conocimiento, Netbiblo, La Coruña, $127 \mathrm{p}$.

MARTÍNEZ PUCHE, A., y VALERO ESCANDELL, J.R. (2000): "Poderes locales y percepción de los problemas medioambientales de la industria. Ejemplos en municipios interiores de Alicante", en GOZÁLVEZ PÉREZ, V. (edit.), Industria y medio ambiente, Universidad de Alicante y AGE, Alicante, pp. 229-243.

MARTÍNEZ PUCHE, A., PRIETO CERDÁN, A., RODRÍGUEZ GUTIÉRREZ, F. (coords.) (2008): Los sistemas locales de empresas y el desarrollo territorial: evolución y perspectivas actuales en un contexto globalizado, Editorial Club Universitario y Departamento de Geografía Humana de la Universidad de Alicante, Alicante, $445 \mathrm{pp}$.

MARTÍNEZ PUCHE, A., PRIETO CERDÁN, A. (2008): "La sostenibilidad integrada y procesos participativos en la provincia de Alicante. Lecciones aprendidas en el marco del proyecto europeo URBACT-MEDINT", en Martínez Puche, A., Salas Arqueros, J.A., Sánchez Navas, J., Cortés Samper, C., (eds.), Sostenibilidad en los espacios rurales. Proyectos europeos, herramientas participativas, experiencias municipales y territoriales en España, publicaciones Universidad de Alicante, Alicante, pp. 227-249.

MONTERO, J.R., FONT, J., TORCAL, M. (eds.) (2006): Ciudadanos, asociaciones y participación en España, Centro de Investigaciones Sociológicas (CIS), Madrid, $430 \mathrm{pp}$.

NOVY, A. y HAMMER, E. (2007): "Radical innovation in the era of liberal governance. The case of Vienna", European Urban and Regional Studies, vol. 14, no 3, pp. 210-222. 
PRATS, F. (1996): "Sostenibilidad y políticas urbanas y locales: el caso de las ciudades españoles", Primer catálogo español de buenas prácticas, vol. I, Ministerio de Obras Públicas, Transporte y Medio Ambiente, Madrid, pp. 91-110.

PRIETO CERDÁN, A. et alii (2009): 12 pasos para la red agenda 21. Nueva metodología para implantar la agenda 21 local, Diputación provincial de Alicante, Alicante, 80 p. 